\title{
MOMENTS OF RANDOM SUMS AND ROBBINS' PROBLEM OF OPTIMAL STOPPING
}

\author{
ALEXANDER GNEDIN, ${ }^{*}$ Utrecht University \\ ALEXANDER IKSANOV, ${ }^{* *}$ National Taras Shevchenko University of Kiev
}

\begin{abstract}
Robbins' problem of optimal stopping is that of minimising the expected rank of an observation chosen by some nonanticipating stopping rule. We settle a conjecture regarding the value of the stopped variable under the rule that yields the minimal expected rank, by embedding the problem in a much more general context of selection problems with the nonanticipation constraint lifted, and with the payoff growing like a power function of the rank.

Keywords: Stopping time; Robbins' problem of minimising the expected rank; Poisson embedding; random sum

2010 Mathematics Subject Classification: Primary 60G40; 60G50
\end{abstract}

Let $X_{1}, \ldots, X_{n}$ be independent random variables sampled sequentially from the uniform $[0,1]$ distribution, and let $Y_{1}<\cdots<Y_{n}$ be their order statistics. The rank $R_{j}$ of the variable $X_{j}$ is defined by setting $R_{j}=k$ on the event $X_{j}=Y_{k}$. Robbins' problem of optimal stopping [3] is that of minimising the expected rank E $R_{\tau}$ over all stopping times $\tau$ that assume values in $\{1, \ldots, n\}$ and are adapted to the natural filtration of the sequence $X_{1}, \ldots, X_{n}$. Let $\tau_{n}$ be the optimal stopping time. The minimum expected rank E $R_{\tau_{n}}$ increases as $n$ grows, and converges to some finite limit $v$ whose exact value is unknown. The closest known upper bound is slightly less than $\frac{7}{3}$. Finding $v$ or even improving the existing rough bounds remains a challenge. A major source of difficulty is that the optimal stopping time $\tau_{n}$ is a very complicated function of the sample. It seems that $\tau_{n}$ has not been computed for $n>3$. Moreover, for large $n$, there is no simplification, and the complexity of the optimal stopping time persists in the ' $n=\infty$ ' limiting form of the problem [6].

In a recent paper Bruss and Swan [4] stressed that it is not even known if $\lim _{\sup } n \mathrm{E} X_{\tau_{n}}$ is finite. They mentioned that the property was first conjectured in [2]. Although the conjecture emerged in connection with attempts to bound $v$ by comparison with the much simpler problem of minimising $\mathrm{E} X_{\tau}$ (or minor variations of the problem), it seems that the question is of independent interest as a relation between the stopped sample value and its rank. In this note we settle the conjecture by proving a considerably more general assertion.

Proposition 1. Fix $p>0$. For $n=1,2, \ldots$, let $\sigma_{n}$ be a random variable with range $\{1, \ldots, n\}$ and arbitrary joint distribution with $X_{1}, \ldots, X_{n}$. Then

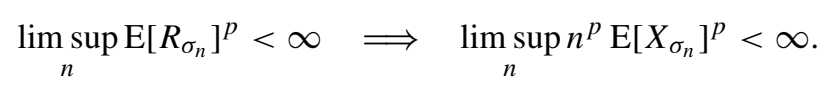

Received 5 July 2011; revision received 24 August 2011.

* Postal address: Department of Mathematics, Utrecht University, Postbus 80010, 3508 TA Utrecht, The Netherlands.

Email address: a.v.gnedin@uu.nl

** Postal address: Faculty of Cybernetics, National Taras Shevchenko University of Kiev, Kiev 01033, Ukraine.

Email address: iksan@univ.kiev.ua 
In particular, $\lim _{n \rightarrow \infty} n^{p} \mathrm{E}\left[X_{\tau_{n}}\right]^{p}<\infty$ for $\tau_{n}$, the stopping time minimising $\mathrm{E}\left[R_{\tau}\right]^{p}$ over all stopping times adapted to $X_{1}, \ldots, X_{n}$.

We defer the proof to the end of the note. The idea is to bound $X_{\sigma_{n}}$ by exploiting properties of a random walk with negative drift.

Let $\xi, \xi_{1}, \xi_{2}, \ldots$ be independent and identically distributed (i.i.d.) nonnegative random variables with $\mu=\mathrm{E} \xi \in(0, \infty)$. Let $S_{k}:=\xi_{1}+\cdots+\xi_{k}$, and, for $\lambda>\mu$, let $M_{\lambda}:=$ $\sup _{k \geq 0}\left(S_{k}-\lambda k\right)$.

Proposition 2. For $p>0$,

$$
\mathrm{E} \xi^{p+1}<\infty \Longleftrightarrow \mathrm{E} M_{\lambda}^{p}<\infty .
$$

Proof. The moment condition on $\xi$ is equivalent to $\mathrm{E}\left[(\xi-\lambda)^{+}\right]^{p+1}<\infty$, and the result follows from Lemma 3.5 of [1].

Corollary 1. Suppose that $\mathrm{E} \xi^{p+1}<\infty$, and let $\sigma$ be a nonnegative integer random variable with $\mathrm{E} \sigma^{p}<\infty$. Then $\mathrm{E} S_{\sigma}^{p}<\infty$.

Proof. The proof follows from $S_{\sigma}^{p} \leq\left(M_{\lambda}+\lambda \sigma\right)^{p} \leq c_{p}\left(M_{\lambda}^{p}+\lambda^{p} \sigma^{p}\right)$, where $c_{p}:=2^{p-1} \vee 1$.

We first apply Corollary 1 to a Poisson embedded, limiting form of the stopping problem with continuous time [6]. Let $\xi_{1}, \xi_{2}, \ldots$ be i.i.d. rate-one exponential variables, let $S_{k}$ be as above, and let $T_{1}, T_{2}, \ldots$ be i.i.d. uniform $[0,1]$ random times, independent of the $\xi_{j}$ s. The points $\left(T_{k}, S_{k}\right)$ are the atoms of a homogeneous planar Poisson process $\mathcal{P}$ in $[0,1] \times[0, \infty)$. To introduce the dynamics, consider an observer whose information at time $t \in[0,1]$ is the (infinite) configuration of points of $\mathcal{P}$ within the strip $[0, t] \times[0, \infty)$, that is, $\left\{\left(T_{k}, S_{k}\right): T_{k} \leq t\right\}$. The rank of point $\left(T_{k}, S_{k}\right)$ is defined as $R_{T_{k}}=k$, meaning that $S_{k}$ is the $k$ th smallest value among $S_{1}, S_{2}, \ldots$. The piece of information added at time $T_{k}$ is the point $\left(T_{k}, S_{k}\right)$, but not the rank $R_{T_{k}}$.

Suppose that the objective of the observer is to minimise $\mathrm{E}\left[R_{\tau}\right]^{p}$ over stopping times $\tau$ that assume values in the random set $\left\{T_{1}, T_{2}, \ldots\right\}$ and are adapted to the information flow of the observer. For the optimal stopping time $\tau_{\infty}$, it is known from the previous studies that $\mathrm{E}\left[R_{\tau_{\infty}}\right]^{p}<\infty$ (see [5] and [6]). Taking $\sigma=R_{\tau_{\infty}}$, we have $\mathrm{E}\left[S_{\sigma}\right]^{p}<\infty$. The case $p=1$ corresponds to the infinite version of Robbins' problem of minimising the expected rank.

Returning to our main task, we wish to apply the above to a finite sample of fixed size. To this end, we shall use the familiar realisation of uniform order statistics through sums of exponential variables, as

$$
\left(Y_{k}, 1 \leq k \leq n\right) \stackrel{\mathrm{D}}{=}\left(\frac{S_{k}}{S_{n}}, 1 \leq k \leq n\right) .
$$

Introducing the event $A_{n}:=\left\{n / S_{n}>1+\varepsilon\right\}$, we can estimate, for $1 \leq k \leq n$, $n^{p} Y_{k}^{p}=n^{p} Y_{k}^{p} \mathbf{1}_{A_{n}}+n^{p} Y_{k}^{p} \mathbf{1}_{A_{n}^{\mathrm{c}}} \leq n^{p} \mathbf{1}_{A_{n}}+(1+\varepsilon)^{p} S_{k}^{p} \leq n^{p} \mathbf{1}_{A_{n}}+c_{p}(1+\varepsilon)^{p}\left(M_{\lambda}^{p}+\lambda^{p} k^{p}\right)$, where we have used $S_{k} \leq M_{\lambda}+\lambda k$. Using a large deviation bound for the probability of $A_{n}$ and sending $\varepsilon \rightarrow 0$, we conclude that, for any random variable $\sigma_{n}$ with values in $\{1, \ldots, n\}$,

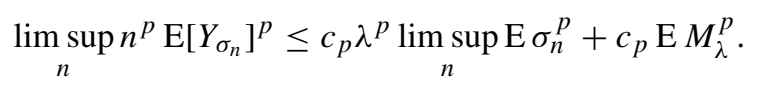


Finally, taking $\sigma_{n}=R_{\tau_{n}}$, Proposition 1 follows from

$$
\limsup _{n} n^{p} \mathrm{E}\left[X_{\tau_{n}}\right]^{p} \leq c_{p} \lambda^{p} \limsup _{n} \mathrm{E}\left[R_{\tau_{n}}\right]^{p}+c_{p} \mathrm{E} M_{\lambda}^{p}<\infty,
$$

since $\mathrm{E}\left[R_{\tau_{n}}\right]^{p}$ converges to a finite limit (see [5] and [6]).

\section{Acknowledgement}

This note was completed during the second author's visit to Utrecht, supported by the Department of Mathematics at Utrecht University and the Dutch stochastic cluster STAR.

\section{References}

[1] Alsmeyer, G. And Iksanov, A. (2009). A log-type moment result for perpetuities and its application to martingales in supercritical branching random walks. Electron. J. Prob. 14, 289-312.

[2] Assaf, D. and Samuel-Cahn, E. (1996). The secretary problem: minimizing the expected rank with i.i.d. random variables. Adv. Appl. Prob. 28, 828-852.

[3] Bruss, F. T. (2005). What is known about Robbins' problem? J. Appl. Prob. 42, 108-120.

[4] Bruss, F. T. AND Swan, Y. C. (2009). A continuous-time approach to Robbins' problem of minimizing the expected rank. J. Appl. Prob. 46, 1-18.

[5] Gianini, J. and Samuels, S. M. (1976). The infinite secretary problem. Ann. Prob. 3, 418-432.

[6] Gnedin, A. V. (2007). Optimal stopping with rank-dependent loss. J. Appl. Prob. 44, 996-1011. 